\title{
Integral Field Spectroscopy of Gravitational Mirages
}

\author{
M.-C. Angonin-Willaime ${ }^{1}$ and Christian Vanderriest ${ }^{2}$
}

\begin{abstract}
This paper deals with applications of bi(tri)dimensional spectroscopy methods to some gravitational mirages. Optical fibre devices are well adapted to the study of the structure and geometry of such phenomena. The improvement that brought bidimensional spectroscopy of few mirages is presented and the need to observe the other candidates is discussed.
\end{abstract}

\section{Introduction}

Gravitational mirages of quasars or AGNs usually present, within a few arcseconds, several point-like images of the source (with possibly very different brightnesses), very close in projection to one or several more-or-less extended galaxies responsible for the phenomenon. It is for such tight and complex structures that integral field spectrography brings a real improvement compared to the other spectrographic methods (Vanderriest and Angonin, 1990). In particular, simultaneously recording the spectra of all the components and being able to make a clean decomposition on reconstructed images has 2 main interests:

1) Study of microlensing (differential magnification of regions with different sizes: emission lines/continuum, continuum/BAL,...);

2) Measurement of the intrinsic characteristics of the components (images, lensing galaxies: nature, redshift, velocity dispersion etc...).

We will illustrate these points by a few examples of gravitational mirages observed with the spectrograph SILFID (Vanderriest and Lemonnier, 1988).

\section{Microlensing effects}

The deflecting galaxy of a gravitational mirage has a "granular" structure: it is composed of stars that can also magnify a (macro-)image in the case of a nearly perfect alignment. This effect is called microlensing. Such an event can be observed in photometry with magnitude variation of the image, but also with spectrography considering that intrinsic structures of the sources that bring different features in the spectrum can be differentially magnified (Rees, 1981; Nemiroff, 1988). The best way to describe a microlensing event is to determine simultaneously the spectra of all the mirage images which is not an easy task without bidimensional spectroscopy.

\footnotetext{
${ }^{1}$ Observatoire de Paris-Meudon (DAEC), France

${ }^{2}$ Canada-France-Hawaii Telescope Corp., Waimea, Hawaii, USA
} 

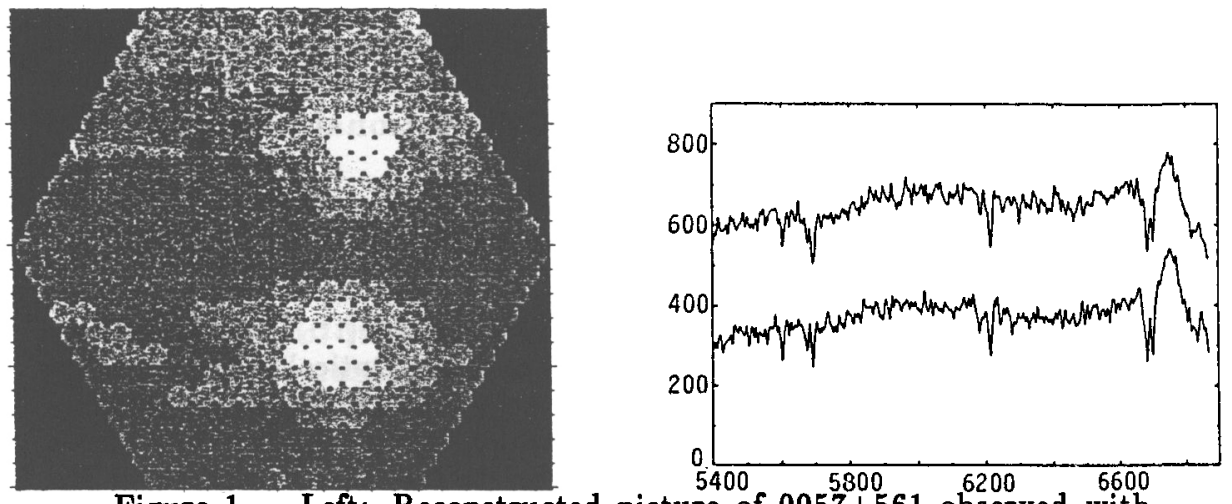

Figure 1. Left: Reconstructed picture of $0957+561$ observed with SILFID in december 1990 . The wavelength range is 4000-7000 $\AA$. Right: Spectra integrated over image A (lower curve) and image B (upper curve) showing differential magnification by microlensing.

Observations with SILFID spectrograph of the first discovered gravitational mirage $0957+561$ (Vanderriest, 1990) in 1990 were done on the CFH telescope. They put in evidence microlensing effect on the $\mathrm{Mg}_{\mathrm{g}} \mathrm{II}$ emission line for image B by comparing the spectra of the two images (Fig.1).

This result is compatible with an amplification ratio between the two images of $\mathrm{A} / \mathrm{B}=0.75$ which has been determined separatly by radio observations.

Another example comes from data obtained with the same spectrograph on the same telescope on the "clover leaf" H1413+117. Spectra of the four images are very similar except in the broad absorption lines of component D (Fig 2; Angonin et al., 1990).

With respect to the other 3 images, the $D$ continuum seems also to be more amplified than the emission lines. All these effects can be explained with differential magnification of source intrinsic regions by microlensing and constrain the shape and the size of the B.A.L. region.

Microlensing is not a rare event. It has been detected for example also in two quadruple quasars: PG $1115+080$ (Angonin et al., in preparation) and 2237+030 (Irwin et al., 1989). To determine characteristics of a gravitational mirage it is necessary to detect microlensing effects and to quantify them. Bidimensional spectrography is the easiest way to reach this information.

\section{Faint components}

The components of gravitational mirages often present different brightnesses. Informations on all of them are needed to model the phenomena especially in aim to measure dark matter of the lens or to determine the Hubble constant (if the time delay between the images is already known). While there is usually no preferential alignment in the mirage configurations, bidimensional spectrography should bring great improvements in the study of such objects. The first advantage is to observe simultaneous spectra of the mirage images to constrain the source nature and confirm the gravitational mirage hypothesis, but the most dif- 

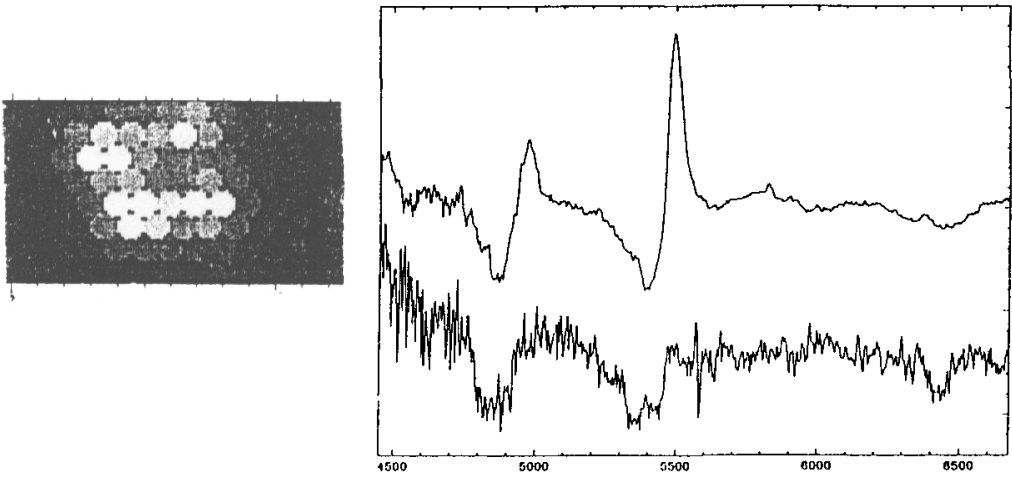

Figure 2. Left: Reconstructed picture of $\mathrm{H} 1413+117$ observed with SILFID in march 1989 . The wavelength range is $4000-7000 \AA$. Right: Normalized spectrum integrated over image $A+B+C$ (upper curve) and residual of the spectrum of image $D$ compared to the previous one (lower curve) to describe the microlensing effects.

ficult challenge is the measurement of the lensing galaxy characteristics: nature, redshift, velocity dispersion,... The galaxy is rather faint and, as an extended object, is often spread over one or more images of the bright quasi-pointlike source. It is then possible to obtain a spectrum by adding all the fibers that contain photons from the fuzzy object and eventually to correct the contribution of the source spectrum by subtracting the spectrum of another image. This problem is critical in compact quadruple quasars like PG $1115+080$ and Q1422+231 (Patnaik et al., 1993) or in the triple starburst galaxy 2016+112 (Langston et al., 1991).

\section{Perspectives}

Bidimensional spectrography is a rewarding technique for almost each and every field of astronomy. But it would be especially useful for extracting key informations inthe tiny crowded fields of several gravitational mirages. Combining high spatial resolution and big photon collector, the new large telescopes will be the best instruments for that field and we hope that many efficient fibre devices that transform a 2-D field into a pseudo-slit like FUEGOS in the ARGUS mode for the VLT will be widespread.

Acknowledgments. M.-C. A.-W. really wants to thank Christian Vanderriest for his talk in Marseille which he had to prepare at the last moment and for his helpful discussions.

\section{Discussion}

N. Douglas: Why invoke microlensing to explain differences between the spectra of the components, instead of uneven absorption along the different paths through the lensing galaxy? 
M.-C. Angonin-Willaime: A differential effect between the continuum and the emission lines, for instance, should imply a surprisingly heterogeneous distribution of the absorbant on a scale comparable to the beam size at the distance of the lens. But, unlikely as it is, this explanation does not even work because we do not observe the expected reddening. This achromatism is incompatible with absorption but is quite characteristic of (micro)lensing events.

Otherwise, it is true that intrinsic variability of the continuum source plus time-delays could mimic the same thing. To be sure, we must compare the spectra of the different images at the same emission time. This was done for $0957+561$ whose time-delay is known and the result is in favour of microlensing. For the other discussed mirages, this is also the most likely explanation because the time-delays are much shorter.

$S$. Dodonov: For the investigation of microlensing effects in $0957+561$, how did you preserve the spectra of the QSO's images from contamination by the (deflecting) galaxy's light?

M.-C. Angonin-Willaime: That is indeed one of the advantages of integral field spectroscopy: in a case like $0957+561$, we can subtract quite accurately the contamination by the galaxy's spectrum. Knowing (from imaging data) the light distribution of the galaxy and having its integrated spectrum over a region outside images $A$ and $B$, we can estimate the galaxy flux under each image, in the areas used for the integration. With the conditions of seeing we had, the correction is typically $4 \%$ in the blue and $10 \%$ in the red for $\mathrm{B}$ and almost negligible for $\mathbf{A}$. This ensures a spectrophotometry for the quasar images better than $1 \%$. Obviously, the correction was a necessity for accurate measurement of the amplification ratios.

\section{References}

Angonin, M-C, Remy, M, Surdej J, Vanderriest, C 1990, A\&A, 233, L4

Irwin, M, Webster, R, Hewett, P, Corrigan, R, Jedrzejewski, R 1989, AJ, 98, 1989

Langston, G, Fisher, J., Aspin, C. 1991, AJ, 102, 1253

Nemiroff, R 1988, ApJ, 335, 593

Patnaik, A, Browne, I, Walsh, D, Chaffee, F, Foltz, C 1992, MNRAS, 259, 1 P

Rees, M 1981, ESO Conference "Scientific importance of high angular resolution at infrared and optical wavelengths", M.H. Ulrich, K. Kjar eds, 423.

Vanderriest, C 1990, Lecture Notes in Physics, 360, 210

Vanderriest, C, Angonin, MC 1992, Lecture Notes in Physics, 406, 97

Vanderriest, C, Lemonnier, JP 1988, in "Instrumentation for ground-based telescopes", proceedings IX Santa Cruz workshop, Robinson eds., Springer publish., 304 